\title{
ANTI-NMDAR AUTOIMMUNE ENCEPHALITIS PRECEEDING SYSTEMIC LUPUS ERYTHEMATOSUS
}

Samara Rosa Sepresse ${ }^{1}$, Danilo Rodrigues Pereira ${ }^{1}$, Lilian Tereza Lavras Costallat ${ }^{1}$, Fernando Cendes $^{1}$, Simone Appenzeller ${ }^{1, *}$

1.Universidade Estadual de Campinas, Campinas (SP), Brazil.

${ }^{*}$ Corresponding author: appenzellersimone@gmail.com

\section{BACKGROUND}

Anti-NMDAR autoimmune encephalitis (AIE) is a disease mediated mainly by NR1 autoantibodies against surface protein epitopes causing reversible neuronal damage. Systemic lupus erythematosus (SLE) is a chronic and multisystemic autoimmune disease that can significantly affect the central nervous system (CNS), mediated primary by NR2 antibodies. We will report a case of a patient with anti-NMDAR autoimmune encephalitis that developed SLE during follow-up period.

\section{CASE REPORT}

A 22-year-old female patient admitted with seizures, dysarthria and chorea. CSF analysis showed increased proteins, and absence of infections. Autoantibody screening revealed anti-NMDA receptor antibody t. With these results, the diagnosis of autoimmune encephalitis was established. Treatment with azathioprine was started, after the end of treatment, the patient evolved with complete clinical remission. One year after the initial treatment, the patient returns to the service with arthritis, malar rash and lymph node enlargement. Serum autoantibody screening revealed antinuclear antibody reagent with coarse speckled nuclear pattern (titer 1/1280); anti-dsDNA positivity and complement consumption. Treatment with methotrexate, prednisone, hydroxychloroquine was started, with complete remission. No CNS additional CNS involvement was noted.

\section{CONCLUSION}

Systemic lupus erythematosus can mimic or be associated with EAl; however, autoantibodies profiles are often distinct. Initially, our patient had only AIE, with no evidence of SLE disease activity. Although the family of anti-glutamate receptor can be found in both diseases, we generally observed distinct subtypes. While NR1 is observed in AIE, NR2 is associated with CNS involvement in SLE. In summary, further studies are needed to follow-up AIE patients to determine the frequency of this association.

\section{KEYWORDS}

Anti-NMDAR autoimmune encephalitis, Systemic lupus erythematosus, Central nervous system. 